\title{
Crystal structure of acid deoxyribonuclease
}

Jan Abendroth ${ }^{1,2}$, Armando Varela-Ramirez ${ }^{3}$, Adrian A. Mejia ${ }^{3}$, Isabelle Q. Phan ${ }^{1,4}$, Donald D. Lorimer $^{1,2}$, Renato J. Aguilera ${ }^{3}$, Thomas E. Edwards ${ }^{1,2}$,

${ }^{1}$ Seattle Structural Genomics Center for Infectious Disease (SSGCID), Seattle, WA 98110 USA ${ }^{2}$ Beryllium Discovery Corp., Bainbridge Island, WA 98110 USA

${ }^{3}$ Department of Biological Sciences, Border Biomedical Research Center, The University of Texas at El Paso, EI Paso, TX 79968 USA

${ }^{4}$ Center for Infectious Disease Research (formerly Seattle Biomedical Research Institute), Seattle, WA 98109 USA

Thomas Edwards (tedwards@be4.com)

Deoxyribonuclease II (DNase II) is also known as acid deoxyribonuclease because it has optimal activity at low $\mathrm{pH}$, which matches the acidic environment of lysosomes where DNase II is typically found in higher eukaryotes. DNase II has also been identified in a few genera of bacteria such as Burkholderia pseudomallei, the causative agent of melioidosis. Recombinant $B$. thailandensis DNase II is highly active at low $\mathrm{pH}$ in the absence of divalent metal ions. The crystal structure of $B$. thailandensis DNase II shows a dimeric quaternary structure which appears capable of binding double-stranded DNA. Each monomer of $B$. thailandensis DNase II exhibits a similar overall fold as numerous phospholipase D (PLD) family members, implying a similar overall catalytic mechanism. We also provide a structural basis for the inhibition of DNase II by Co2+ ions, in contrast to other nuclease which require divalent cations. The structural and biochemical data presented here provide insights into the atomic structure and catalytic mechanism of the DNase II family of enzymes. 